\title{
Damped harmonic oscillator: pure states of the bath and exact master equations
}

\author{
Andrey Pereverzev $⿴$ \\ Department of Chemistry, Trinity University, San Antonio, TX 78212
}

(Dated: March 29, 2019)

\begin{abstract}
Time evolution of a harmonic oscillator linearly coupled to a heat bath is compared for three classes of initial states for the bath modes - grand canonical ensemble, number states and coherent states. It is shown that for a wide class of number states the behavior of the oscillator is similar to the case of the equilibrium bath. If the bath modes are initially in coherent states, then the variances of the oscillator coordinate and momentum, as well as its entanglement to the bath, asymptotically approach the same values as for the oscillator at zero temperature and the average coordinate and momentum show a Brownian-like behavior. We derive an exact master equation for the characteristic function of the oscillator valid for arbitrary factorized initial conditions. In the case of the equilibrium bath this equation reduces to an equation of the Hu-Paz-Zhang type, while for the coherent states bath it leads to an exact stochastic master equation with a multiplicative noise.
\end{abstract}

PACS numbers: 05.30.-d, 05.40.-a, 02.50.-r

\section{INTRODUCTION}

The model of an oscillator linearly coupled to the bath of harmonic oscillator has played an important part in statistical mechanics [1, 2, 3], quantum optics [4, 5] and quantum measurement theory 6 , 7, 8 ]. In most studies of this model the initial state for the whole system is taken as a mixed density matrix. In particular, one often uses factorized initial state where the bath modes are in thermal equilibrium and the oscillator is in a pure state. Less often the so-called thermal initial conditions are used 9, 10, 11, 12. . Pure states of the bath are rarely considered 13], except for the vacuum state of the bath.

Our goal in this paper is to compare the behavior of the oscillator for different pure initial states for the bath modes to the case of the bath in equilibrium. The quantities we will be considering are the averages and variances of the oscillator coordinate and momentum, as well as $\operatorname{Tr} \rho^{2}$ as a measure of oscillator entanglement to the bath. We also would like to show how different initial states for the bath modes lead to different exact master equations for the oscillator density matrix. In deriving such equations we will use an exact formal solution for the characteristic function of the oscillator, rather then the path integral techniques for the reduced density matrix 7, 10, 11, 14. This approach makes it possible for this model to obtain master equations for arbitrary factorized initial conditions.

This paper is organized as follows. In Sec. II we consider the model and its exact solution. In Sections III IV and $\nabla$ the oscillator behavior is considered, respectively, for the bath modes in equilibrium, number states and coherent states. Exact master equations are discussed in VI] Concluding remarks are given in Sec. VII

*Electronic address: andrey.pereverzev@trinity.edu

\section{THE MODEL OF A LINEARLY COUPLED OSCILLATOR}

The system Hamiltonian is given by

$$
\begin{aligned}
H= & \hbar \nu a^{\dagger} a+\sum_{k} \hbar \omega_{k} b_{k}^{\dagger} b_{k}+\sum_{k} \hbar u_{k} a^{\dagger} b_{k} \\
& +\sum_{k} \hbar u_{k}^{*} b_{k}^{\dagger} a+\sum_{k} \hbar v_{k} a^{\dagger} b_{k}^{\dagger}+\sum_{k} \hbar v_{k}^{*} b_{k} a .
\end{aligned}
$$

Here $a^{\dagger}$ and $a$ are the creation and annihilation operators of the harmonic oscillator, and $b_{k}^{\dagger}$ and $b_{k}$ are the creation and annihilation operators for the bath modes. The coefficients are assumed to be such that the Hamiltonian is a positive definite quadratic form. The coordinate and momentum operators for the oscillator are related to $a^{\dagger}$ and $a$ through

$$
x=\sqrt{\frac{\hbar}{2 m \nu}}\left(a^{\dagger}+a\right), \quad p=i \sqrt{\frac{\hbar m \nu}{2}}\left(a^{\dagger}-a\right),
$$

where $m$ is the oscillator mass. By a suitable choice of coefficients Hamiltonian (11) reduces to the Hamiltonian with coordinate-coordinate coupling or the rotating wave approximation (RWA) Hamiltonian. In particular, in the latter case the last two terms in (10) are dropped. We will assume that if the number of the bath modes increases to infinity the frequency $\omega_{k}$ becomes a continuous function of $k$. We will refer to the limit of the infinite number of modes with average energy of each mode being held constant as the thermodynamic limit.

Various forms of Hamiltonian (11) corresponding to different choices of frequencies and coupling constants as well as its exact diagonalization has been extensively studied in the literature. General but formal discussion of the diagonalization of a Hermitian quadratic bosonic form [of which (10) is a special case] can be found in [15]. Systems of oscillators with coordinate only coupling were considered in [16]. Detailed investigation of Hamiltonian 
(11) in the case of coordinate coupling can be found in references 2, 9, 17. Relation between several forms of (1) and the translationally invariant Hamiltonian with coordinate coupling was discussed in [18]. Relation between the coordinate coupling and RWA is studied in [12, 17].

The equations of motion for the annihilation and creation operators are

$$
\begin{aligned}
& \dot{a}=-i \nu a-i \sum_{k} u_{k} b_{k}-i \sum_{k} v_{k} b_{k}^{\dagger}, \\
& \dot{b}_{k}=-i \omega_{k} b_{k}-i u_{k}^{*} a-i v_{k} a^{\dagger}, \\
& \dot{a^{\dagger}}=i \nu a^{\dagger}+i \sum_{k} u_{k}^{*} b_{k}^{\dagger}+i \sum_{k} v_{k}^{*} b_{k}, \\
& \dot{b}_{k}^{\dagger}=i \omega_{k} b_{k}^{\dagger}+i u_{k} a^{\dagger}+i v_{k}^{*} a .
\end{aligned}
$$

This system of equations can be solved subject to the set of initial conditions $a(0)=a, b_{k}(0)=b_{k}, a^{\dagger}(0)=a^{\dagger}$, and $b_{k}^{\dagger}(0)=b_{k}^{\dagger}$. Since system (3) is linear, its solutions will depend linearly on the initial conditions. In particular, $a(t)$ is given by

$$
a(t)=A(t) a+\sum_{k} B_{k}(t) b_{k}+C(t) a^{\dagger}+\sum_{k} D_{k}(t) b_{k}^{\dagger} .
$$

Similarly, for $a^{\dagger}(t)$ we have

$$
a^{\dagger}(t)=A^{*}(t) a^{\dagger}+\sum_{k} B_{k}^{*}(t) b_{k}^{\dagger}+C^{*}(t) a+\sum_{k} D_{k}^{*}(t) b_{k} .
$$

Coefficients $A(t), B_{k}(t), C(t)$, and $D_{k}(t)$ satisfy the following relation (see [15] for details):

$$
|A(t)|^{2}-|C(t)|^{2}+\sum_{k}\left|B_{k}(t)\right|^{2}-\sum_{k}\left|D_{k}(t)\right|^{2}=1 .
$$

They can, in principle, be calculated for a each particular form of frequencies and coupling parameters. For the purposes of this paper we will not need explicit expressions for these coefficients. The only assumptions we will use is that in the thermodynamic limit coefficients $A(t)$ and $C(t)$ vanish for $t \rightarrow \infty$, and $B_{k}(t)$ and $D_{k}(t)$ remain bounded in the same limit. Physically these requirements correspond to the fact the the initial state of the oscillator is forgotten for long times while any observables associated with it (e.g., average energy) remain finite. The detailed calculations of coefficients that show such behavior as well as conditions on the coupling constants and frequencies in the thermodynamic limit can be found in the original references [1, 2, , 9, 10, 17].

The reduced dynamics of the oscillator is conveniently described in terms of a symmetrically ordered characteristic function defined as [4]

$$
\chi(\eta, t)=\operatorname{Tr}\left(\rho e^{\eta a^{\dagger}-\eta^{*} a}\right) .
$$

Here $\chi(\eta, t)$ can be treated as a function of $\eta, \eta^{*}$ and $t$. We will always suppress the second variable to simplify the notation. Using characteristic function (7) we can calculate expectation values of the symmetrized products of operators $a^{\dagger}$ and $a$. It can also be converted to any of the quasi-probability distribution functions or the reduced density matrix [4]. We will also use $\operatorname{Tr} \rho^{2}$ as a measure of purity of the oscillator state $\rho$. In terms of the characteristic function $\operatorname{Tr} \rho^{2}$ is given by

$$
\operatorname{Tr} \rho^{2}=\frac{1}{\pi} \int d^{2} \eta|\chi(\eta)|^{2}
$$

To see how $\chi(\eta, t)$ evolves in time we use the Heisenberg picture and insert expressions (44) and (5) into (7) to obtain

$$
\chi(\eta, t)=\operatorname{Tr}\left(\rho e^{\left(\eta A^{*}-\eta^{*} C\right) a^{\dagger}-\left(\eta^{*} A-\eta C^{*}\right) a} \prod_{k} e^{\left(\eta B_{k}^{*}-\eta^{*} D_{k}\right) b_{k}^{\dagger}-\left(\eta^{*} B_{k}-\eta D_{k}^{*}\right) b_{k}}\right) .
$$

The time dependence of the coefficients is suppressed here and whenever possible to avoid heavy notation.

In this paper we consider only factorized initial conditions, i.e., we assume that the initial density matrix of the whole system $\rho_{\text {total }}$ factorizes into the oscillator and bath density matrices as $\rho_{\text {total }}=\rho \otimes \rho_{\text {bath }}$. In this case the characteristic function takes the form

$$
\chi(\eta, t)=\chi\left(\left(\eta A^{*}-\eta^{*} C\right), 0\right) F(\eta, t),
$$

with

$$
F(\eta, t)=\operatorname{Tr}\left(\rho_{b a t h} \prod_{k} e^{\left(\eta B_{k}^{*}-\eta^{*} D_{k}\right) b_{k}^{\dagger}-\left(\eta^{*} B_{k}-\eta D_{k}^{*}\right) b_{k}}\right) .
$$

Equation (10) expresses the oscillator characteristic function at time $t$ in terms of the initial characteristic function. In the limit of long times, when $A$ and $C$ vanish, characteristic function $\chi(\eta, t)$ is determined by the asymptotic form of $F(\eta, t)$.

We will now consider the time evolution and asymp- 
totic values of the average oscillator coordinate and momentum, the coordinate and momentum variances and $\operatorname{Tr} \rho^{2}$ for different initial states of the bath.

\section{EQUILIBRIUM STATE OF THE BATH}

This section consists primarily of an overview of wellknown results. The state of the bath is the grand canonical ensemble given by

$$
\rho_{e q}=\prod_{k}\left(1-e^{-\beta \hbar \omega_{k}}\right) e^{-\beta \hbar \omega_{k} b_{k}^{\dagger} b_{k}} .
$$

Using the identity

$$
\operatorname{Tr}\left(\rho_{e q} e^{\eta b_{k}^{\dagger}-\eta^{*} b_{k}}\right)=e^{-|\eta|^{2}\left(\bar{n}_{k}+\frac{1}{2}\right)}
$$

with

$$
\bar{n}_{k}=\frac{1}{e^{\beta \hbar \omega_{k}}-1}
$$

we find function $F(\eta, t)$ to be

$$
F(\eta, t)=e^{-\alpha|\eta|^{2}+\gamma^{*} \eta^{2}+\gamma \eta^{* 2}} .
$$

Here coefficients $\alpha$ and $\gamma$ are given by

$$
\alpha=\sum_{k}\left(\left|B_{k}\right|^{2}+\left|D_{k}\right|^{2}\right)\left(\bar{n}_{k}+\frac{1}{2}\right),
$$

$$
\gamma=\sum_{k} B_{k} D_{k}\left(\bar{n}_{k}+\frac{1}{2}\right)
$$

We will often use coefficients $\alpha$ and $\gamma$ in the limits $t \rightarrow \infty$ or $T \rightarrow 0$. In such cases we will use the notation $\alpha_{\infty}, \gamma_{\infty}$ and $\alpha^{0}, \gamma^{0}$, respectively. If both limits are taken we will use $\alpha_{\infty}^{0}$ and $\gamma_{\infty}^{0}$.

The time evolution of the average coordinate and momentum is easily calculated either through the characteristic function, or by directly using (4) and (5).

$$
\begin{aligned}
& \langle x(t)\rangle=\sqrt{\frac{\hbar}{2 m \nu}}\left(\left(A^{*}+C\right)\left\langle a^{\dagger}\right\rangle+\left(A+C^{*}\right)\langle a\rangle\right), \\
& \langle p(t)\rangle=i \sqrt{\frac{\hbar m \nu}{2}}\left(\left(A^{*}+C\right)\left\langle a^{\dagger}\right\rangle-\left(A+C^{*}\right)\langle a\rangle\right)
\end{aligned}
$$

There is no dependence on the state of the bath. For arbitrary temperature $\langle x(t)\rangle$ and $\langle p(t)\rangle$ depend only on their initial average values. In the limit of infinitely long times $\langle x(t)\rangle$ and $\langle p(t)\rangle$ vanish.

For the variances of the oscillator coordinate and momentum we obtain

$$
\begin{aligned}
\left\langle x^{2}(t)\right\rangle-\langle x(t)\rangle^{2}= & \frac{\hbar}{2 m \nu}\left[\left(A^{*}+C\right)^{2}\left(\left\langle a^{\dagger} a^{\dagger}\right\rangle-\left\langle a^{\dagger}\right\rangle\left\langle a^{\dagger}\right\rangle\right)+\left(A+C^{*}\right)^{2}(\langle a a\rangle-\langle a\rangle\langle a\rangle)\right. \\
& \left.+2\left|\left(A+C^{*}\right)\right|^{2}\left(\left\langle a^{\dagger} a\right\rangle-\left\langle a^{\dagger}\right\rangle\langle a\rangle+\frac{1}{2}\right)+2\left(\alpha+\gamma+\gamma^{*}\right)\right], \\
\left\langle p^{2}(t)\right\rangle-\langle p(t)\rangle^{2}= & \frac{\hbar m \nu}{2}\left[-\left(A^{*}-C\right)^{2}\left(\left\langle a^{\dagger} a^{\dagger}\right\rangle-\left\langle a^{\dagger}\right\rangle\left\langle a^{\dagger}\right\rangle\right)-\left(A-C^{*}\right)^{2}(\langle a a\rangle-\langle a\rangle\langle a\rangle)\right. \\
& \left.+2\left|\left(A-C^{*}\right)\right|^{2}\left(\left\langle a^{\dagger} a\right\rangle-\left\langle a^{\dagger}\right\rangle\langle a\rangle+\frac{1}{2}\right)+2\left(\alpha-\gamma-\gamma^{*}\right)\right] .
\end{aligned}
$$

These quantities depend both on the initial state of the oscillator and the temperature of the bath. For $t \rightarrow \infty$ we have

$$
\begin{aligned}
\left\langle x^{2}\right\rangle-\langle x\rangle^{2} & =\frac{\hbar}{m \nu}\left(\alpha_{\infty}+\gamma_{\infty}+\gamma_{\infty}^{*}\right), \\
\left\langle p^{2}\right\rangle-\langle p\rangle^{2} & =\hbar m \nu\left(\alpha_{\infty}-\gamma_{\infty}-\gamma_{\infty}^{*}\right) .
\end{aligned}
$$

Coefficients $\alpha_{\infty}$ and $\gamma_{\infty}$ are proportional to $\bar{n}_{k}$. Therefore, at high temperatures variances (19) grow as $k T$.

The measure of the oscillator purity is given by the integral:

$\operatorname{Tr} \rho^{2}=\frac{1}{\pi} \int d^{2} \eta\left|\chi\left(\left(\eta A^{*}-\eta^{*} C\right), 0\right)\right|^{2} e^{-2\left(\alpha|\eta|^{2}-\gamma^{*} \eta^{2}-\gamma \eta^{* 2}\right)}$.

This integral can be calculated for specific initial states of the oscillator. For infinitely long times, when the initial state is forgotten, we obtain

$$
\operatorname{Tr} \rho^{2}=\frac{1}{2 \sqrt{\alpha_{\infty}^{2}-4\left|\gamma_{\infty}\right|^{2}}} .
$$

For high temperatures $\operatorname{Tr} \rho^{2}$ is proportional to $1 / k T$. The state of the oscillator becomes less pure as the temperature grows. Let us note that for intermediate times $\operatorname{Tr} \rho^{2}$ 
can take lower values than its value at $t \rightarrow \infty$. For zero temperature of the bath the asymptotic value of $\operatorname{Tr} \rho^{2}$ will, in general, be less then one since the oscillator remains dressed at $T=0$.

For the special case of the RWA Hamiltonian, coefficients $C(t)$ and $D_{k}(t)$ in (4) and (5) are equal to zero. As a result, $\alpha_{\infty}^{0}=\frac{1}{2}$ and $\gamma=0$ and for long times $\operatorname{Tr} \rho^{2}=1$. In this case the reduced vacuum of the oscillator is a pure state that is identical to the ground state of the uncoupled oscillator.

\section{THE NUMBER STATES FOR THE BATH MODES}

We now consider the initial state of the bath with each mode in a number state.

$$
\left|\left\{n_{k}\right\}\right\rangle=\left|n_{k_{1}}\right\rangle \otimes\left|n_{k_{2}}\right\rangle \otimes \cdots
$$

with $\left\{n_{k}\right\}$ denoting a set of occupation numbers $n_{k}$ for all modes.
Function $F(\eta, t)$ can be calculated using the identity 19]

$$
\left\langle n_{k}\left|e^{\eta b_{k}^{\dagger}-\eta^{*} b_{k}}\right| n_{k}\right\rangle=e^{-\frac{|\eta|^{2}}{2}} L_{n_{k}}\left(|\eta|^{2}\right)
$$

where $L_{n}(x)$ is a Laguerre polynomial. We obtain

$$
\begin{aligned}
F(\eta, t)= & e^{-\alpha^{0}|\eta|^{2}+\gamma^{0 *} \eta^{2}+\gamma^{0} \eta^{* 2}} \\
& \times \prod_{k} L_{n_{k}}\left(\left|\left(\eta B^{*}{ }_{k}-\eta^{*} D_{k}\right)\right|^{2}\right) .
\end{aligned}
$$

The behavior of average coordinate and momentum of the oscillator is exactly the same as for the equilibrium state of the bath and given by (17). Thus, $\langle x(t)\rangle$ and $\langle p(t)\rangle$ do not depend on the particular set of occupation numbers. Both quantities vanish for $t \rightarrow \infty$. For the variances of the coordinate and momentum we have

$$
\begin{aligned}
\left\langle x^{2}(t)\right\rangle-\langle x(t)\rangle^{2}= & \frac{\hbar}{2 m \nu}\left[\left(A^{*}+C\right)^{2}\left(\left\langle a^{\dagger} a^{\dagger}\right\rangle-\left\langle a^{\dagger}\right\rangle\left\langle a^{\dagger}\right\rangle\right)+\left(A+C^{*}\right)^{2}(\langle a a\rangle-\langle a\rangle\langle a\rangle)\right. \\
& \left.+2\left|\left(A+C^{*}\right)\right|^{2}\left(\left\langle a^{\dagger} a\right\rangle-\left\langle a^{\dagger}\right\rangle\langle a\rangle+\frac{1}{2}\right)+2\left(\tilde{\alpha}+\tilde{\gamma}+\tilde{\gamma}^{*}\right)\right], \\
\left\langle p^{2}(t)\right\rangle-\langle p(t)\rangle^{2}= & \frac{\hbar m \nu}{2}\left[-\left(A^{*}-C\right)^{2}\left(\left\langle a^{\dagger} a^{\dagger}\right\rangle-\left\langle a^{\dagger}\right\rangle\left\langle a^{\dagger}\right\rangle\right)-\left(A-C^{*}\right)^{2}(\langle a a\rangle-\langle a\rangle\langle a\rangle)\right. \\
& \left.+2\left|\left(A-C^{*}\right)\right|^{2}\left(\left\langle a^{\dagger} a\right\rangle-\left\langle a^{\dagger}\right\rangle\langle a\rangle+\frac{1}{2}\right)+2\left(\tilde{\alpha}-\tilde{\gamma}-\tilde{\gamma}^{*}\right)\right],
\end{aligned}
$$

where

$$
\begin{aligned}
& \tilde{\alpha}=\sum_{k}\left(\left|B_{k}\right|^{2}+\left|D_{k}\right|^{2}\right)\left(n_{k}+\frac{1}{2}\right), \\
& \tilde{\gamma}=\sum_{k} B_{k} D_{k}\left(n_{k}+\frac{1}{2}\right) .
\end{aligned}
$$

The variances differ from the equilibrium ensemble case by the replacement of $\bar{n}_{k}$ with $n_{k}$.

To get a better picture of how these values relate to the equilibrium case we have to make some assumption about the occupation numbers. Let us consider an ensemble of the occupation number states corresponding to the number state decomposition of the equilibrium density matrix

$$
\rho_{e q}=\sum_{\left\{n_{k}\right\}}\left|\left\{n_{k}\right\}\right\rangle P_{n}\left(\left\{n_{k}\right\}\right)\left\langle\left\{n_{k}\right\}\right| .
$$

Here the probability for a particular set of occupation numbers $P_{n}\left(\left\{n_{k}\right\}\right)$ is given by

$$
P_{n}\left(\left\{n_{k}\right\}\right)=\prod_{k}\left(1-e^{-\beta \hbar \omega_{k}}\right) e^{-\beta \hbar \omega_{k} n_{k}} .
$$

We now assume that the number states are taken from ensemble (27). Any quantities calculated for each individual number state (e.g., averages, variances, $\operatorname{Tr} \rho^{2}$ ) can then be averaged over $P_{n}\left(\left\{n_{k}\right\}\right)$ to obtain their average values in ensemble (27). These latter averages will give typical values for the pure state quantities in ensemble (27).

Averaging variances (25) over ensemble (27) will give the same variances as for the equilibrium case. In particular, in the limit of long times we have

$$
\begin{aligned}
& \overline{\left\langle x^{2}\right\rangle-\langle x\rangle^{2}}=\frac{\hbar}{m \nu}\left(\alpha_{\infty}+\gamma_{\infty}+\gamma_{\infty}^{*}\right), \\
& \overline{\left\langle p^{2}\right\rangle-\langle p\rangle^{2}}=\hbar m \nu\left(\alpha_{\infty}-\gamma_{\infty}-\gamma_{\infty}^{*}\right) .
\end{aligned}
$$

Here we use overlining to denote averaging over the ensemble of pure states. 
The fact that the average variances are the same as for the equilibrium ensemble is not surprising since expressions in (25) are linear in $n_{k}$ 's. More importantly, in the thermodynamic limit almost all states in ensemble (27) will have the same variances as in equilibrium. Let us show this for the coordinate variance. We can treat the coordinate variance as a function of random variables $n_{k}$ described by the distribution $P_{n}\left(\left\{n_{k}\right\}\right)$. Calculating the variance of this function for the distribution $P_{n}\left(\left\{n_{k}\right\}\right)$ we obtain.

$$
\begin{aligned}
& \overline{\left(\left\langle x^{2}\right\rangle-\langle x\rangle^{2}\right)^{2}}-\overline{\left(\left\langle x^{2}\right\rangle-\langle x\rangle^{2}\right)} \cdot \overline{\left(\left\langle x^{2}\right\rangle-\langle x\rangle^{2}\right)} \\
& =\frac{\hbar^{2}}{m^{2} \nu^{2}} \sum_{k}\left|\left(B_{k}+D_{k}^{*}\right)\right|^{4}\left(\overline{n_{k}^{2}}-\bar{n}_{k}^{2}\right) .
\end{aligned}
$$

We note that coefficients $B_{k}$ and $D_{k}$ must depend on the number of bath modes $N$ as $1 / \sqrt{N}$ in order for the quantities like (25) to remain finite in the thermodynamic limit. Therefore the sum over $k$ in (30) is proportional to $1 / N$ and vanishes for $N \rightarrow \infty$. A similar argument can be applied to $\left(\left\langle p^{2}(t)\right\rangle-\langle p(t)\rangle^{2}\right)$. Thus, as in equilibrium case, we expect the variances to grow as $k T$ for high occupation numbers.

Let us now consider the behavior of $\operatorname{Tr} \rho^{2}$. Using definition (8) and characteristic function (24) we obtain

$$
\operatorname{Tr} \rho^{2}=\frac{1}{\pi} \int d^{2} \eta\left|\chi\left(\left(\eta A^{*}-\eta^{*} C\right), 0\right)\right|^{2} e^{-2\left(\alpha^{0}|\eta|^{2}-\gamma^{0 *} \eta^{2}-\gamma^{0} \eta^{* 2}\right)} \prod_{k} L_{n_{k}}^{2}\left(\left|\left(\eta B^{*}{ }_{k}-\eta^{*} D_{k}\right)\right|^{2}\right) .
$$

If number states are taken from the ensemble (27) we can calculate average $\operatorname{Tr} \rho^{2}$ for number states in this ensemble. Using the identity [20]

$$
\sum_{n} L_{n}^{2}(x) z^{n}=\frac{1}{1-z} \exp \left(-\frac{2 z x}{1-z}\right), \quad(|z|<1)
$$

we obtain

$\overline{\operatorname{Tr} \rho^{2}}=\frac{1}{\pi} \int d^{2} \eta\left|\chi\left(\left(\eta A^{*}-\eta^{*} C\right), 0\right)\right|^{2} e^{-2\left(\alpha|\eta|^{2}-\gamma^{*} \eta^{2}-\gamma \eta^{* 2}\right)}$.

This is exactly the same as $\operatorname{Tr} \rho^{2}$ in (20). We can see that, at least on the average, $\operatorname{Tr} \rho^{2}$ for number states from ensemble (27) is the same at all times as for the case of equilibrium bath. As a consequence, in the limit of long times the state of the oscillator becomes less pure for higher occupation numbers for the bath modes.

\section{COHERENT STATES FOR THE BATH MODES}

We now consider the case where all bath modes are initially in coherent states.

$$
\left|\left\{\beta_{k}\right\}\right\rangle=\left|\beta_{k_{1}}\right\rangle \otimes\left|\beta_{k_{2}}\right\rangle \otimes \cdots
$$

with $\left\{\beta_{k}\right\}$ denoting a set of complex numbers $\beta_{k}$ specifying the coherent states. One can interpret such a state as the most classical state of the bath. Function $F(\eta, t)$ is calculated to be

$$
F(\eta, t)=e^{\delta^{*} \eta-\delta \eta^{*}-\alpha^{0}|\eta|^{2}+\gamma^{0 *} \eta^{2}+\gamma^{0} \eta^{* 2}} .
$$

Here

$$
\delta=\sum_{k}\left(B_{k} \beta_{k}+D_{k} \beta_{k}^{*}\right)
$$

For the average coordinate and momentum we obtain

$$
\begin{aligned}
\langle x(t)\rangle= & \sqrt{\frac{\hbar}{2 m \nu}}\left(\left(A^{*}+C\right)\left\langle a^{\dagger}\right\rangle+\left(A+C^{*}\right)\langle a\rangle\right. \\
& \left.+\delta+\delta^{*}\right), \\
\langle p(t)\rangle= & i \sqrt{\frac{\hbar m \nu}{2}}\left(\left(A^{*}-C\right)\left\langle a^{\dagger}\right\rangle-\left(A-C^{*}\right)\langle a\rangle\right. \\
& \left.+\delta^{*}-\delta\right) .
\end{aligned}
$$

To get a better understanding of how $\langle x(t)\rangle$ and $\langle p(t)\rangle$ behave we have to make some assumption about parameters $\beta_{k}$.

Analogously to the procedure used for the number state bath let us assume that the coherent states are taken from the ensemble corresponding to the coherent states decomposition of the equilibrium density matrix

$$
\rho_{e q}=\int d^{2}\left\{\beta_{k}\right\}\left|\left\{\beta_{k}\right\}\right\rangle P_{c}\left(\left\{\beta_{k}\right\}\right)\left\langle\left\{\beta_{k}\right\}\right| .
$$

The probability distribution $P_{c}\left(\left\{\beta_{k}\right\}\right)$ is given by

$$
P_{c}\left(\left\{\beta_{k}\right\}\right)=\prod_{k} \frac{1}{\pi \bar{n}_{k}} \exp \left(\frac{-\left|\beta_{k}\right|^{2}}{\bar{n}_{k}}\right) .
$$

Decomposition (38) is just the $P$-representation of the equilibrium density matrix [4].

If the coherent states are taken from ensemble (38), then, for each set of the coherent states, $\delta(t)$ is a realization of a complex colored normal noise with zero mean and correlation functions given by

$$
\begin{gathered}
\overline{\delta(t) \delta^{*}\left(t^{\prime}\right)}=\sum_{k}\left(B_{k}(t) B_{k}^{*}\left(t^{\prime}\right)+D_{k}(t) D_{k}^{*}\left(t^{\prime}\right)\right) \bar{n}_{k}, \\
\overline{\delta(t) \delta\left(t^{\prime}\right)}=\sum_{k}\left(B_{k}(t) D_{k}\left(t^{\prime}\right)+B_{k}\left(t^{\prime}\right) D_{k}(t)\right) \bar{n}_{k} .
\end{gathered}
$$


Clearly, $\langle x(t)\rangle$ and $\langle p(t)\rangle$ are also realizations of the normal noise. The mean in this case is, in general, non-zero but will go to zero for long times.

Let us evaluate the typical asymptotic values taken by $\langle x(t)\rangle$ and $\langle p(t)\rangle$. This can be done by calculating average $\langle x\rangle^{2}$ and $\langle p\rangle^{2}$ for ensemble (38).

$$
\begin{aligned}
& \overline{\langle x\rangle^{2}}=\frac{\hbar}{m \nu}\left(\alpha_{\infty}+\gamma_{\infty}+\gamma_{\infty}^{*}-\alpha_{\infty}^{0}-\gamma_{\infty}^{0}-\gamma_{\infty}^{0}{ }^{*}\right), \\
& \overline{\langle p\rangle^{2}}=\hbar m \nu\left(\alpha_{\infty}-\gamma_{\infty}-\gamma_{\infty}^{*}-\alpha_{\infty}^{0}+\gamma_{\infty}^{0}+\gamma_{\infty}^{0}{ }^{*}\right) .
\end{aligned}
$$

For high temperatures these quantities are proportional to $k T$. Therefore typical asymptotic values for $\langle x\rangle$ and $\langle p\rangle$ are proportional to $\sqrt{k T}$.

The coordinate and momentum variances are the same as for the case of equilibrium bath at zero temperature, i.e., they are given by (18) with $\alpha$ and $\gamma$ replaced by $\alpha^{0}$ and $\gamma^{0}$, respectively. Therefore, these variances do not depend on a particular set of parameters $\beta_{k}$ and their asymptotic values are the same as for the reduced vacuum state of the oscillator.

The value of $\operatorname{Tr} \rho^{2}$ is given by

$$
\operatorname{Tr} \rho^{2}=\frac{1}{\pi} \int d^{2} \eta\left|\chi\left(\left(\eta A^{*}-\eta^{*} C\right), 0\right)\right|^{2} e^{-2\left(\alpha_{0}|\eta|^{2}-\gamma_{0}^{*} \eta^{2}-\gamma_{0} \eta^{* 2}\right)},
$$

and is the same as in the case of zero temperature bath with the asymptotic value given by

$$
\operatorname{Tr} \rho^{2}=\frac{1}{2 \sqrt{\left(\alpha_{\infty}^{0}\right)^{2}-4\left|\gamma_{\infty}^{0}\right|^{2}}} .
$$

More generally, the asymptotic characteristic function is determined by the asymptotic form of function $F(\eta, t)$ (35) and corresponds to the density matrix that is a displaced reduced vacuum state $\rho_{v a c}$,

$$
\rho=D(\delta) \rho_{v a c} D^{\dagger}(\delta),
$$

where the displacement operator $D(\delta)$ is given by

$$
D(\delta)=e^{\delta a^{\dagger}-\delta^{*} a} .
$$

We can conclude that for coherent states bath in the limit of long times the oscillator becomes localized in the following sense: the coordinate and momentum variances, as well as entanglement to the bath, are same as for the reduced vacuum (and do not depend on temperature), while the average coordinate and momentum randomly fluctuate with the amplitude of the fluctuations proportional to $\sqrt{k T}$. The rate of such localization is of the order of the relaxation rate as can be concluded from (18) for $T=0$.

For the special case of the RWA Hamiltonian the asymptotic state of the oscillator is a pure coherent state. The oscillator can start in a mixed or pure state, it then goes through a period of entanglement and asymptotically its state becomes a pure coherent state with randomly fluctuating average coordinate and momentum. If the oscillator is initially in a coherent state it will always remain in a coherent state, never entangling with the bath [21]. In Appendix we give explicit expression for $\operatorname{Tr} \rho^{2}(t)$ for two types of initial oscillator states for the RWA case.

\section{EXACT MASTER EQUATIONS}

There is a similarity of a mathematical nature between the coherent states bath and bath in equilibrium. In both cases function $F(\eta, t)$ is Gaussian, and, as a result, the characteristic function satisfies simple master equations as will be shown shortly.

Before doing so let us note that for any factorized initial conditions (for any system-bath model) there always exists an exact equation for the reduced density matrix with the time evolution governed by a time-dependent operator that does not depend on the state of the system. Obtaining an explicit expression for such an operator can, in general, be a difficult task. Let us show that such an operator can be constructed for the present model. We will continue to use the characteristic function space because of its mathematical convenience. If necessary the equations can be transformed into other representations. To simplify the demonstration we introduce the following transformed characteristic function

$$
\tilde{\chi}(\eta, t)=\frac{\chi(\eta, t)}{F(\eta, t)} .
$$

Using expression (10) for $\chi(\eta, t)$ and the fact that $\tilde{\chi}(\eta, 0)=\chi(\eta, 0)$ we can write function $\tilde{\chi}(\eta, t)$ at time $t$ in terms of the $\tilde{\chi}(\eta, 0)$ as

$$
\tilde{\chi}(\eta, t)=\tilde{\chi}\left(\left(\eta A^{*}-\eta^{*} C\right), 0\right) .
$$

We now use the fact that $\tilde{\chi}(\eta, t)$ depends on $\eta, \eta^{*}$ and $t$ only through $\left(\eta A^{*}-\eta^{*} C\right)$ and $\left(\eta^{*} A-\eta C^{*}\right)$. Differentiating solution (48) with respect to time we have

$$
\begin{aligned}
\frac{\partial \tilde{\chi}}{\partial t}= & \frac{\partial \tilde{\chi}}{\partial\left(\eta A^{*}-\eta^{*} C\right)}\left(\eta \dot{A}^{*}-\eta^{*} \dot{C}\right) \\
& +\frac{\partial \tilde{\chi}}{\partial\left(\eta^{*} A-\eta C^{*}\right)}\left(\eta^{*} \dot{A}-\eta \dot{C}^{*}\right) .
\end{aligned}
$$


For derivatives with respect to $\eta$ and $\eta^{*}$ we obtain

$$
\begin{aligned}
& \frac{\partial \tilde{\chi}}{\partial \eta}=\frac{\partial \tilde{\chi}}{\partial\left(\eta A^{*}-\eta^{*} C\right)} A^{*}-\frac{\partial \tilde{\chi}}{\partial\left(\eta^{*} A-\eta C^{*}\right)} C^{*}, \\
& \frac{\partial \tilde{\chi}}{\partial \eta^{*}}=-\frac{\partial \tilde{\chi}}{\partial\left(\eta A^{*}-\eta^{*} C\right)} C+\frac{\partial \tilde{\chi}}{\partial\left(\eta^{*} A-\eta C^{*}\right)} A .
\end{aligned}
$$

Solving the last two equations for the derivatives of $\tilde{\chi}$ with respect to $\left(\eta A^{*}-\eta^{*} C\right)$ and $\left(\eta^{*} A-\eta C^{*}\right)$ and substituting them in (49) we obtain a closed equation for $\tilde{\chi}$ :

$$
\frac{\partial \tilde{\chi}}{\partial t}=\left(\xi^{*}(t) \eta+\zeta(t) \eta^{*}\right) \frac{\partial \tilde{\chi}}{\partial \eta}+\left(\xi(t) \eta^{*}+\zeta^{*}(t) \eta\right) \frac{\partial \tilde{\chi}}{\partial \eta^{*}} .
$$

Here $\xi(t)$ and $\zeta(t)$ are given by

$$
\xi(t)=\frac{A^{*} \dot{A}-C^{*} \dot{C}}{|A|^{2}-|C|^{2}}, \quad \zeta(t)=\frac{C \dot{A}-A \dot{C}}{|A|^{2}-|C|^{2}}
$$

Substituting definition of $\tilde{\chi}$ (47) into equation (51) we obtain the following equation for $\chi$

$$
\begin{aligned}
\frac{\partial \chi}{\partial t}= & \left(\xi^{*}(t) \eta+\zeta(t) \eta^{*}\right)\left(\frac{\partial \chi}{\partial \eta}-\left(\frac{\partial \ln F}{\partial \eta}\right) \chi\right)+\left(\xi(t) \eta^{*}+\zeta^{*}(t) \eta\right)\left(\frac{\partial \chi}{\partial \eta^{*}}-\left(\frac{\partial \ln F}{\partial \eta^{*}}\right) \chi\right) \\
& +\left(\frac{\partial \ln F}{\partial t}\right) \chi
\end{aligned}
$$

This is a closed equation for characteristic function $\chi(\eta, t)$. The explicit form of the time-dependent operator is determined by function $F(\eta, t)$, which, in turn, is determined by the initial state of the bath. We will not go into the analysis of this equation and limit ourselves to a few remarks. This equation is always local in the $\eta$-space. However, transforming it into the quasiprobability distribution space or coordinate or momentum representation for the density matrix will, in general, lead to non-local equations. Only for some special forms of $F(\eta, t)$ can we expect to get local equations in these representations. Note that exact master equations for the oscillator-bath model that appeared in the literature use equilibrium initial state for the bath [2, 7], squeezed equilibrium for the bath [14], or modified equilibrium for the whole system [10, 11]. When converted into the characteristic function space all these equations contain time dependent operators that are at most quadratic in $\eta, \eta^{*}$, $\partial / \partial \eta$, and $\partial / \partial \eta^{*}$. This form of the time dependent operator is related to the fact that propagating function for such initial conditions is Gaussian. Inspection of equation (53) shows that such a simple form of the time dependent operator in our case is possible only for Gaussian functions $F(\eta, t)$.

We now consider particular form of equation (53) for this special case, i.e., when function $F(\eta, t)$ is given by

$$
F(\eta, t)=e^{\underline{\delta}^{*} \eta-\underline{\delta} \eta^{*}-\underline{\alpha}|\eta|^{2}+\underline{\gamma}^{*} \eta^{2}+\underline{\gamma} \eta^{* 2} .}
$$

Here $\underline{\delta}, \underline{\alpha}$ and $\underline{\gamma}$ are time dependent parameters characterizing each particular Gaussian state of the bath. Function (54) will include such states for the bath modes as equilibrium, squeezed states, squeezed and displaced equilibrium, etc. Substituting (54) into (53) we obtain after some simplifications

$$
\begin{aligned}
\frac{\partial \chi}{\partial t}= & \left(\xi^{*}(t) \eta+\zeta(t) \eta^{*}\right) \frac{\partial \chi}{\partial \eta}+\left(\xi(t) \eta^{*}+\zeta^{*}(t) \eta\right) \frac{\partial \chi}{\partial \eta^{*}} \\
& +\kappa(t)|\eta|^{2} \chi+\mu^{*}(t) \eta^{2} \chi+\mu(t) \eta^{* 2} \chi \\
& +\sigma^{*}(t) \eta \chi-\sigma(t) \eta^{*} \chi
\end{aligned}
$$

Coefficients $\kappa(t), \mu(t)$ and $\sigma(t)$ are given by

$$
\begin{aligned}
& \kappa(t)=\underline{\alpha}\left(\xi+\xi^{*}\right)-2\left(\zeta \underline{\gamma}^{*}+\zeta^{*} \underline{\gamma}\right)-\underline{\dot{\alpha}}, \\
& \mu(t)=\zeta \underline{\alpha}-2 \xi \underline{\gamma}+\underline{\dot{\gamma}}, \\
& \sigma(t)=\zeta \underline{\delta^{*}}-\xi \underline{\delta}+\underline{\dot{\delta}} .
\end{aligned}
$$

In the case of the equilibrium bath we need to put $\underline{\alpha}=$ $\alpha, \underline{\gamma}=\gamma$ and $\underline{\delta}=0$ in (56). In this case the last two terms on the right-hand side of equation (55) disappear and the equation becomes the characteristic function version of the Hu-Paz-Zhang equation 2, 7] for the Hamiltonian (1), i. e., it will allow for the possibility of momentummomentum and momentum-coordinate coupling between the bath and the oscillator.

If the bath modes are initially in coherent states then we have to use $\underline{\alpha}=\alpha^{0}, \underline{\gamma}=\gamma^{0}$ and $\underline{\delta}=\delta$. If the coherent states are taken from ensemble (38), then, for each particular set of states, $\sigma(t)$ will be a realization of a complex colored normal noise. The equation becomes a stochastic master equation with a multiplicative noise. In this case (55) describes the oscillator localization in the sense discussed above with the average coordinate and momentum subject to random fluctuation. Equations of such type can be of interest in the theory of 
quantum measurement as an alternative to the stochastic Schrödinger equations [22]. In particular, compared to the latter equations, equation (55) does not conserve purity of the state. Such behavior is more physically plausible for a system coupled to a bath.

\section{CONCLUDING REMARKS}

Equilibrium density matrix is often considered as an irreducible concept, viz., it is assumed that this is a true state of the bath in each individual experiment. We believe that such a view is an oversimplification, and it is more realistic to assume that true state of the bath is a density matrix or even a pure state which includes some random component. Ensemble of such states for different realizations of the random component gives an equilibrium density matrix. Such a view is partially supported by classical statistical mechanics. Indeed, most physicists agree that the "true state" of the classical bath in thermal equilibrium is a point in phase space. The coarse-grained macroscopic observables for such a point (e.g., number of particles in a volume element) are essentially the same as calculated for one of the Gibbs ensembles (of such points) for large enough course-graining [23].

It is true that the ensemble decomposition of the equilibrium density matrix in terms of other density matrices (or pure states) is not unique, and that the averages calculated for any decomposition are identical to equilibrium averages. We believe, however, that it can be of importance, that, when quantities like variances and $\operatorname{Tr} \rho^{2}$ are calculated for individual members of the ensemble, we can get drastically different behavior for the system for different decompositions, as was shown in this paper.

\section{ACKNOWLEDGMENTS}

The author would like to thank Dr. Gonzalo Ordóñez for fruitful discussions. Part of this research was supported by grants from the Robert A. Welch Foundation (W-1442) and the Petroleum Research Fund, administered by the American Chemical Society.

\section{APPENDIX}

In this Appendix we give explicit expressions for $\operatorname{Tr} \rho^{2}(t)$ for two types of initial pure states of the oscillator for the RWA Hamiltonian if the bath modes are initially in coherent states.

Let the oscillator be initially in a squeezed state obtained by acting on the oscillator ground state with the squeezing and displacement operators

$$
|\psi\rangle=D(\alpha) S(\varepsilon)|0\rangle
$$

Here the squeezing operator $S(\varepsilon)$ is given by

$$
S(\varepsilon)=e^{\frac{\varepsilon^{*}}{2} a-\frac{\varepsilon}{2} a^{\dagger}},
$$

and $\varepsilon=r e^{2 i \phi}$ is a complex squeezing parameter. Using expression (43) for $\operatorname{Tr} \rho^{2}$ we obtain

$$
\operatorname{Tr} \rho^{2}(t)=\frac{1}{\sqrt{1+4|A(t)|^{2}\left(1-|A(t)|^{2}\right) \sinh ^{2} r}}
$$

The maximum entanglement [or minimum $\operatorname{Tr} \rho^{2}(t)$ is reached when $|A(t)|^{2}=1 / 2$. Moreover, $\operatorname{Tr} \rho^{2}(t)$ is a symmetric function of $|A(t)|^{2}$ with respect to this point. When $|A(t)|^{2}=1 / 2$ we have

$$
\operatorname{Tr} \rho^{2}=\frac{1}{\sqrt{1+\sinh ^{2} r}}
$$

We have larger entanglement for larger squeezing with $\operatorname{Tr} \rho^{2}$ going to zero when $r$ goes to infinity.

We now consider an initial state of the oscillator given by a superposition of two coherent states

$$
|\psi\rangle=\frac{1}{\sqrt{N}}(|\alpha\rangle+|\beta\rangle)
$$

where $N$ is the normalization constant,

$$
N=2+\langle\alpha \mid \beta\rangle+\langle\beta \mid \alpha\rangle
$$

In this case we obtain for $\operatorname{Tr} \rho^{2}(t)$

$$
\begin{aligned}
\operatorname{Tr} \rho^{2}(t)= & 1+\frac{2}{N^{2}}\left(e^{-\left(1-|A(t)|^{2}\right)|\alpha-\beta|^{2}}+e^{-|A(t)|^{2}|\alpha-\beta|^{2}}\right. \\
& \left.-e^{-|\alpha-\beta|^{2}}-1\right) .
\end{aligned}
$$

One can easily verify that $\operatorname{Tr} \rho^{2}(t)$ takes its minimum value again at $|A(t)|^{2}=1 / 2$, and $\operatorname{Tr}^{2}(t)$ is again a symmetric function of $|A(t)|^{2}$ with respect to that point, which may be a general property of this model. Let us consider $\operatorname{Tr}^{2}(t)$ at $|A(t)|^{2}=1 / 2$. We have

$$
\operatorname{Tr} \rho^{2}=1-\frac{2}{N^{2}}\left(1-e^{-\frac{|\alpha-\beta|^{2}}{2}}\right)
$$

Using (A.6), writing $\langle\alpha \mid \beta\rangle$ as $R e^{i \varphi}$ and remembering that

$$
e^{-\frac{|\alpha-\beta|^{2}}{2}}=|\langle\alpha \mid \beta\rangle|
$$

we obtain

$$
\operatorname{Tr} \rho^{2}=1-\frac{(1-R)^{2}}{2(1+R \cos \varphi)^{2}}
$$

We can see from the last expression that the minimum $\operatorname{Tr} \rho^{2}$ decreases with the decreasing overlap between the two coherent states. Note, however, that in this case $\operatorname{Tr} \rho^{2}$ can never become less then $1 / 2$. 
[1] A. O. Caldeira and A. J. Leggett, Physica A 121, 587 (1983).

[2] F. Haake and R. Reibold, Phys. Rev. A 32, 2462 (1985).

[3] H. Grabert, P. Schramm, and G. L. Ingold, Phys. Rep 168, 115 (1988).

[4] D. F. Walls and G. J. Milburn, Quantum Optics (Springer-Verlag, Berlin, 1994).

[5] H. Carmichael, An Open System Approach to Quantum Optics (Springer-Verlag, Berlin, 1993).

[6] W. G. Unruh and W. H. Zurek, Phys. Rev. D 40, 1071 (1989).

[7] B. L. Hu, J. P. Paz, and Y. Zhang, Phys. Rev. D 45, 2843 (1992).

[8] D. Guilini, E. Joos, C. Kiefer, J. Kupsch, I. O. Stomatescu, and H. D. Zeh, Decoherence and the Appearance of a Classical World in Quantum Theory (Springer, Berlin, 1996).

[9] V. Hakim and V. Ambegaokar, Phys. Rev. A 32, 423 (1985).

[10] R. Karrlein and H. Grabert, Phys. Rev. E 55, 153 (1997).

[11] L. D. Romero and J. P. Paz, Phys. Rev. A 55, 4070 (1997).

[12] M. R. daCosta, A. O. Caldeira, S. M. Dutra, and
H. Westfahl, Phys. Rev. A 61, 022107 (2000).

[13] T. Petrosky and V. Barsegov, Phys. Rev. E 65, 046102 (2002).

[14] B. L. Hu and A. Matacz, Phys. Rev. D 49, 6612 (1994).

[15] N. N. Bogoliubov and N. N. Bogoliubov, Jr., An Introduction to Quantum Statistical Mechanics (Gordon and Breach Science Publishers, Philadelphia, 1994).

[16] G. W. Ford, M. Kac, and P. Mazur, J. Math. Phys. 6, 504 (1965).

[17] K. Lindenberg and B. J. West, Phys. Rev. A 30, 568 (1984).

[18] G. W. Ford, J. T. Lewis, and R. F. O'Connell, Phys. Rev. A 37, 4419 (1988).

[19] K. E. Cahill and R. J. Glauber, Phys. Rev. 177, 1857 (1969).

[20] I. S. Gradshteyn and I. M. Ryzhik, Tables of Integrals, Series, and Products (Academic Press, New York, 1980).

[21] R. J. Glauber, Phys. Lett. 21, 650 (1966).

$[22]$ U. Weiss, Quantum Dissipative Systems (World Scientific, Singapore, 1999), 2nd ed.

[23] J. L. Lebowitz, Physica A 194, 1 (1993). 